\title{
Separator design optimization for collecting the finely dispersed particles from the gas flows
}

\author{
Vadim E. Zinurov ${ }^{1, *}$, Oksana S. Popkova ${ }^{1}$, and $V u$ L. Nguyen ${ }^{1}$ \\ ${ }^{1}$ Kazan State Power Engineering University, 420066, Kazan, Krasnoselskaya str., 51, Russia
}

\begin{abstract}
This paper deals with the problem of gas flow purification from the finely dispersed particles, which is becoming significantly important nowadays due to the growth of production capacities. The authors developed a separator in order to solve this problem. This paper shows the design scheme of device and the principle of its operation. The authors studied the changes in the pressure losses within the device at different inlet rate of gas flow, as well as different parameters of finely dispersed particles: density and size. The studies show that in order to improve the quality of gas flow purification from the finely dispersed particles, it is necessary to increase the energy costs due to an increase in the pressure loses in the separator.
\end{abstract}

\section{Introduction}

The tightened up-to-date requirements to the purity of process emissions lead to the search for new technologies to purify the air from the finely dispersed particles smaller than 10 $\mu \mathrm{m}$. According to the World Health Organization, the fine particles smaller than $10 \mu \mathrm{m}$, contained in the atmospheric air, are much more harmful to the human body than the large particles. The finely dispersed particles of up to $10 \mu \mathrm{m}$, due to the size, easily penetrate into the human lungs and settle there. These particles are especially dangerous for the employees of enterprises, which emit the contaminated gas flows, containing the finely dispersed particles, into the environment. Also, the inhabited areas, located near such enterprises, are in the greatest danger. In this regard, the purification of gas flow from the finely dispersed particles smaller than $10 \mu \mathrm{m}$ is one of the most relevant issues [1-8].

At the moment, the purification of gas flows from various particles at enterprises is carried out by a wide range of devices: inertial dust collectors, cyclones, battery cyclones, electrostatic filters, wet devices, bag filters, etc. The choice of devices to purify the gas flows from solid particles depends on a large number of factors: required purification degree, thermal, electrostatic and abrasive properties of particles, etc. [9]. It should be noted that very often the choice of devices to purify the air depends on the economic capabilities of enterprise. Therefore, the majority of enterprises use relatively inexpensive devices, which include various modifications of dust collection chambers, cyclones, etc. [10-13]. The advantages of such devices are the following: simplicity of operation, lack of mechanisms, low cost, etc. However, one of the main drawbacks is the low degree of gas

\footnotetext{
*Corresponding author: vadd 93@mail.ru
} 
flow purification from the particles smaller than $10 \mu \mathrm{m}$, which is below 5-10\%. An increase in the degree of purification of gas flows from the solid particles is achieved by creation of process line that requires significant economic investments. As a rule, such line consists of several coarse purification devices: dust collection chambers and cyclones, which allow to purify the gas flows efficiently from the particles larger than $10 \mu \mathrm{m}$. After that another, more expensive and complex device is used, such as bag filter or electrostatic filter, which allows to perform the final purification of gas flow from the finely dispersed particles smaller than $10 \mu \mathrm{m}$ with efficiency of more than $95 \%$.

This scheme is quite well implemented at enterprises. However, the rapid contamination of bag or electrostatic filter leads to a significant reduction in the degree of purification of gas flows from the finely dispersed particles smaller than $10 \mu \mathrm{m}$. In order to solve this problem, more frequent replacement of expensive elements of bag filters or cleaning of electrostatic filters is required that is an unfavourable factor for all enterprises. It should be noted that the installation of bag filter is about 1.5-2 times cheaper than an electric filter, but the operation is more expensive [14]. This is especially true for the small enterprises, where frequent replacement of filter elements is a measure, difficult to implement from a financial point of view, as the replacement of filter elements or cleaning of devices causes the production process stops. As a result, the finely dispersed particles smaller than $10 \mu \mathrm{m}$ are almost completely emitted into the environment after the process cycles. In this regard, the development and searching for new process engineering solutions, that can increase the degree of purification of gas flows from the finely dispersed particles smaller than $10 \mu \mathrm{m}$, is a quiet relevant task [15-20].

\section{Description of device}

In order to increase the degree of purification of gases from the finely dispersed particles of up to $10 \mu \mathrm{m}$, and to reduce the economic costs for maintenance of corresponding devices, the authors developed a separator that allows to collect the particles with a diameter of less than $10 \mu \mathrm{m}$ with an efficiency of more than $50 \%$.

This separator (Fig. 1) consists of 5 rows of arc-shaped elements, arranged in a trapezoidal case. The arc-shaped elements are arranged in the separator at an angle of $30^{\circ}$ to ensure the equal flow.

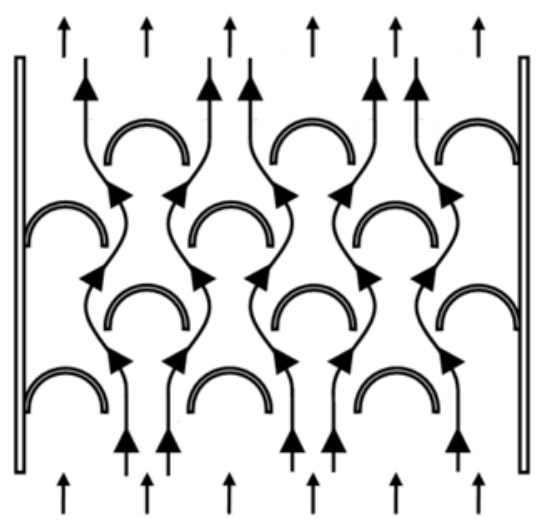

Fig. 1. The simplified 2D-model of separator (top view perpendicular to the arc-shaped elements).

The purification of dusty gas flow from the finely dispersed particles is carried out as follows: the gas enters the separator through the inlet nozzle of special gas duct, then when the gas flows around the arc-shaped elements inside of device, centrifugal forces arise and significantly affect the flow structure, and, as a result, the particles are pushed out from the 
structured flow and adhere to the walls of arc-shaped elements or fall to the bottom of device. After that the purified gas exits the separator through the outlet nozzle. Due to the fact that the gas purification from the finely dispersed particles is carried out mainly due to the centrifugal forces, then in order to achieve the maximum value, the elements are arranged against each other as per the following concept: a circle, drawn from the center of any arc-shaped element, must pass through the extreme points of adjacent rows. The simplified model of device is shown in Fig. 1.

This device is proposed to be used as an intermediate stage for the gas purification from the finely dispersed particles smaller than $10 \mu \mathrm{m}$, which will be located after the coarse cleaning devices prior to the bag or electrostatic filter. Thus, the separator will extend the service life of expensive filters by 2 or more times.

\section{Purpose of this study}

The purpose of this paper is to study the changes in the pressure losses within the device at different inlet rate values of gas flow and the parameters of finely dispersed particles: density and size.

In the course of previous studies, several models of separators with the inlet section sizes from $0.1 \times 0.1$ to $0.4 \times 0.4 \mathrm{~m}^{2}$ were considered by means of numerical simulation. In the course of this numerical simulation, the efficiency of device was changed within the range of $0.01-2.4 \mathrm{~m}^{3} / \mathrm{s}$, as well as the diameters of particles were changed from 1 to $15 \mu \mathrm{m}$ and the rate at the inlet nozzle of separator was also changed from 1 to $20 \mathrm{~m} / \mathrm{s}$. It should be noted that during the numerical simulation, the adhesion condition was set for all the surfaces of elements. On the basis of studies, the following equations were obtained:

$$
\begin{aligned}
& E=1-0.4598 \mathrm{e}^{-1.21 \mathrm{Stk}}, \\
& E_{0}=0.5-0.4006 \mathrm{e}^{-0.0327 \mathrm{Stk}}, \\
& E_{1}=1-0.7945 \mathrm{e}^{-0.6304 \mathrm{Stk}},
\end{aligned}
$$

where $E$ - purification efficiency of gas flow from the finely dispersed dust particles, $E_{0}-$ efficiency of the first two rows of separator, $E_{1}$ - efficiency of one purification stage, Stk the Stokes number.

In order to calculate the operational efficiency of separator, the following formula was used:

$$
E=1-\frac{n_{k}}{n}
$$

where $n_{k}$ - number of particles, remained in the gas flow after its purification in the separator, $n$ - number of particles in the gas flow before purification.

Efficiency of the first two rows $E_{0}$ was determined as follows:

$$
E_{0}=\frac{n_{0}}{n}
$$

where $n_{0}$ - number of particles, remained in the first two rows of arc-shaped separator elements.

The efficiency of purification stage $E_{1}$ was determined by the following formula: 


$$
E_{1}=1-\left(\frac{1-E}{1-E_{0}}\right)^{\frac{1}{m-2}}
$$

where $m$ - number of rows of arc-shaped elements in the separator.

It should be noted that the Eq. (5) was introduced in order to evaluate the efficiency of gas flow purification from the finely dispersed particles by the first two rows due to the fact that the purification within this area is carried out mainly on account of a sharp change in the flow direction. After the gas passes through two rows of arc-shaped elements, the movement of the gas flow acquires a structure due to the influence of centrifugal forces on the gas. Therefore, the purification degree concept was introduced. A row of arc-shaped elements is the purification stage itself, where the gas flow is cleaned under the influence of centrifugal forces, i.e. the rows, located after the first two rows. This purification stage is determined by the Eq. (6).

In the course of previous studies, it was found that the calculated coefficient of hydraulic resistance of device is $\xi=3.883$.

The Stokes number was determined by the following formula:

$$
\mathrm{Stk}=\frac{\rho_{a} a^{2} W_{h}}{\mu h},
$$

where $\rho_{a}$ - density of particles, $\mathrm{kg} / \mathrm{m}^{3} ; a$ - diameter of particles, $\mathrm{m} ; W_{h}$ - rate within the narrowing parts of separator, $\mathrm{m} / \mathrm{s}, \mu-$ gas dynamic viscosity coefficient, $\mathrm{Pa} \cdot \mathrm{s} ; h$ - radius of circles around the elements, $\mathrm{m}$.

On the basis of obtained Eq. (1)-(7), the change in pressure losses within the separator was studied at different inlet rates of gas flow. It should be noted that in order to obtain the data, allowing to choose the optimum technological and geometrical dimensions of the separator for highly efficient purification of gas flow from the finely dispersed particles with low hydraulic resistance, the following parameters were varied: diameter of particles and density within the range of $1-10 \mu \mathrm{m}$ and $700-7000 \mathrm{~kg} / \mathrm{m}^{3}$, respectively. This range of particle sizes is due to the fact that the devices of the first stage for rough purification, purify the gas from them with low efficiency. The chosen density is the most typical for the dispersed phase of particles in the dusty gas flows, emitted by industrial enterprises. Moreover, the efficiency of gas purification $\mathrm{E}$ from the finely dispersed particles was set within the range of $0.9-0.99$, as well as the inlet gas flow rate $W$ was set within the range of 4-20 m/s. In order to obtain the dependencies, one of the parameters was changed, and the rest were taken as constant values. The constant parameters: particle diameter $-4 \mu \mathrm{m}$, density of particles $-1000 \mathrm{~kg} / \mathrm{m}^{3}$ and gas purification efficiency -0.95 . Also in the course of study, the following thermophysical and physical parameters were taken as constant: gas dynamic viscosity coefficient $-\mu=18.1 \cdot 10^{-6} \mathrm{~Pa} \cdot \mathrm{s}$, gas flow density $-\rho=1.28 \mathrm{~kg} / \mathrm{m}^{3}$ and volume flow rate $-Q=0.31 \mathrm{~m}^{3} / \mathrm{s}$.

In the course of study, based on the changing and constant parameters, the dimensions of inlet section, the diameter of arc-shaped elements and the number of elements in the row were calculated. The inlet section of the separator has a square shape.

The side of inlet section of separator, $\mathrm{mm}$ was determined by the following formula:

$$
B=\sqrt{\frac{Q}{W}},
$$

where $Q$ - volume gas flow rate, $\mathrm{m}^{3} / \mathrm{s}$.

The diameter the arc-shaped elements $b, \mathrm{~mm}$ was calculated by the following formula: 


$$
b=\frac{B}{2 n_{b}}
$$

where $n_{b}$ - number of arc-shaped elements in a row.

The pressure losses in the separator were determined by the following formula:

$$
\Delta P=\xi \frac{H}{h} \frac{\rho W^{2}}{2},
$$

where $H$ - distance between the first and last rows of arc-shaped elements, $\mathrm{m}$.

\section{Results of studies and clarification of them}

The results of studies are shown graphically in Fig. 2-4. The numerical studies show that the higher the requirements to the gas flow purification from the finely dispersed particles, the higher the energy costs of corresponding process. The pressure losses in the separator during the gas flow purification increase upon the following factors: with a decrease in the particles density (Fig. 2) and their diameters (Fig. 3), as well with an increase in the efficiency of gas flow purification (Fig. 4).

The increase in the particles density leads to a decrease in the pressure losses within the device, due to the fact that with an increase in the density of particles, the purification of gas flow from them is simplified, since with constant particles size an increase in density of them leads to an increase in weight of them. The higher the weight of particles, the easier to push them out of the structured dusty flow when the gas moves around the arc-shaped elements (Fig. 2).

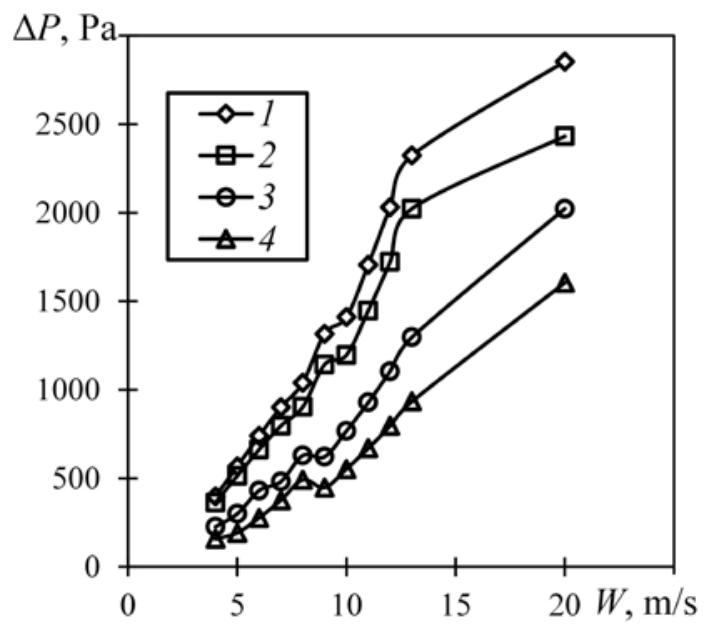

Fig. 2. Dependency of change in the pressure losses in the separator on the inlet gas flow rate at different values of particles density, $\mathrm{kg} / \mathrm{m}^{3}: 1-700 ; 2-1000 ; 3-3000 ; 4-7000$.

The results of studies show that, on average, when the gas flow rate is within the range of 4 to $20 \mathrm{~m} / \mathrm{s}$, the pressure losses in the separator are equal to $1273,1100,733$ and $541 \mathrm{~Pa}$ at particles density of $700,1000,3000$ and $7000 \mathrm{~kg} / \mathrm{m}^{3}$, respectively. It is worth noting that an increase in the inlet rate of gas flow leads to an increase in the pressure losses in the device. When the inlet gas flow rate is equal to $4-5 \mathrm{~m} / \mathrm{s}$, the pressure losses in the separator are on average equal to $482,438,262$ and $174 \mathrm{~Pa}$ for the particles with a density of 700 , 
1000,3000 and $7000 \mathrm{~kg} / \mathrm{m}^{3}$, respectively. An increase in the inlet gas flow rate up to 6-9 $\mathrm{m} / \mathrm{s}$ leads to an increase in the pressure losses in the separator, on average, to $999,875,540$ and $397 \mathrm{~Pa}$ at the particles density of $700,1000,3000$ and $7000 \mathrm{~kg} / \mathrm{m}^{3}$, respectively. At higher gas flow rates of $10-15 \mathrm{~m} / \mathrm{s}$, the pressure losses in the separator, on average, are equal to $1867,1597,1024$ and $737 \mathrm{~Pa}$ for the particles with density of $700,1000,3000$ and $7000 \mathrm{~kg} / \mathrm{m}^{3}$, respectively. Thus, the analysis of parameters of particles, in particular, their density, contained in the dusty gas flows, can significantly affect the reduction of energy costs. The possibility to choose the optimum inlet rate of gas flow will significantly reduce the pressure losses in the separator (Fig. 2).

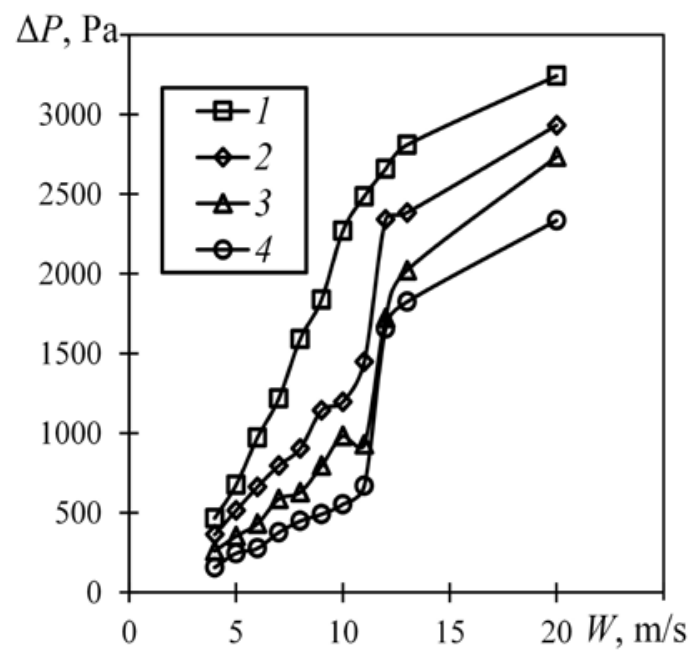

Fig. 3. Dependency of change in the pressure losses in the separator on the inlet gas flow rate at different values of diameter of particles, $\mu \mathrm{m}: 1-2 ; 2-4 ; 3-6 ; 4-10$.

The results of studies show that, on average, when the gas flow rate is within the range of $4-20 \mathrm{~m} / \mathrm{s}$, the pressure losses in the separator are equal to $1684,1223,953$ and $752 \mathrm{~Pa}$ at the diameter of particles equal to 2, 4, 6 and $10 \mu \mathrm{m}$, respectively. As can be seen in Fig. 2, the inlet rate of gas flow has the greatest impact on the pressure losses. When the rates are low, the pressure losses are also low, but an increase in the rate leads to a significant increase in the pressure losses in the separator. Thus, when the inlet rate of gas flow is $4-5$ $\mathrm{m} / \mathrm{s}$, the pressure losses in the separator on average are equal to $570,438,306$ and $201 \mathrm{~Pa}$ for the particles with a diameter of 2, 4, 6 and $10 \mu \mathrm{m}$, respectively. An increase the gas rate up to $6-9 \mathrm{~m} / \mathrm{s}$ leads to an increase in the pressure losses on average to $1403,875,609$ and $397 \mathrm{~Pa}$ when the diameter of particles is equal to 4,6 and $10 \mu \mathrm{m}$, respectively. When the inlet gas flow rates is $10-15 \mathrm{~m} / \mathrm{s}$, an increase in the pressure losses in the separator to 2554 , 1506,1077 and $737 \mathrm{~Pa}$ is observed, at the diameter of particles equal to 2, 4, 6 and $10 \mu \mathrm{m}$, respectively (Fig. 3).

An increase in the efficiency of gas flow purification from the finely dispersed particles leads to an increase in the pressure losses in the separator. Thus, when the inlet gas flow rate is within the range of $4-20 \mathrm{~m} / \mathrm{s}$, the pressure losses in the separator are, on average, equal to 969,1097 and $1453 \mathrm{~Pa}$ with the gas purification efficiency of $0.9,0.95$ and 0.99 , respectively. When the inlet gas flow rate is up to $10 \mathrm{~m} / \mathrm{s}$, the pressure losses, on average, are equal to 605,729 and $997 \mathrm{~Pa}$ with the gas purification efficiency of $0,9,0,95$ и 0,99 respectively. When the rate is within the range of $10-15 \mathrm{~m} / \mathrm{s}$, then the pressure losses are equal to 1310,1506 and $2132 \mathrm{~Pa}$ and the efficiency is equal to $0,9,0,95$ и 0,99 respectively (Fig. 4). 


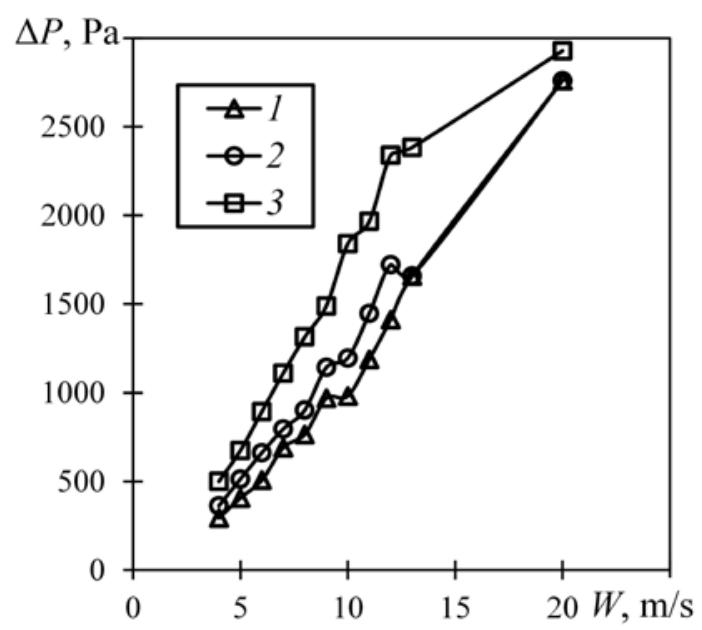

Fig. 4. Dependency of change in the pressure losses in the separator on the inlet gas flow rate at different values of purification of dusty gas flow from the finely dispersed particles: $1-0.9 ; 2-0.95$; $3-0.99$.

Thus, the conducted studies show that in order to minimize the energy costs, required for the process of gas flow purification from the finely dispersed particles, the optimum inlet rate of gas flow is equal to $10 \mathrm{~m} / \mathrm{s}$ and less.

\section{Conclusion}

The obtained Eq. (1)-(10) allow us to demonstrate by calculation that the pressure losses in the separator vary significantly, depending on the inlet rate of gas flow, the density of particles and their diameter. The studies show that the use of separator in the technological scheme of gas purification from the finely dispersed particles after the coarse purification devices can provide the gas purification with an efficiency of not less than 0.9 from the particles with a diameter of less than $10 \mu \mathrm{m}$ and with pressure loses in the apparatus of not more than 1000-1450 Pa. The advantages of separator are the following: simplicity of design, lack of moving mechanisms, low cost, high efficiency of gas flow purification from the finely dispersed particles.

This study was funded by RFBR according to the research project No. 19-07-01188.

\section{References}

1. I. Jakstoniene, S. Konoverskyte, P. Vaitiekunas, Užvalgos, 2(3), 141-149 (2009)

2. S.M. Clifford, J. Zhang, T. Sigsgaard, M. Jantunen, P.J. Lioy, R. Samson, M.H. Karol, Environ. Health Perspect., 115, 958-964 (2007)

3. G. Husken, M. Hunger, H.J. Brouwers, Building and environment, 44, 12, 2463-2474 (2009)

4. A.V. Dmitriev, V.E. Zinurov, O.S. Dmitrieva, MATEC Web Conf., 224, 02073 (2018)

5. D. Muenhor, Environment international, 36, 7, 690-698 (2010)

6. A.V. Dmitriev, V.E. Zinurov, O.S. Dmitrieva, IOP Conf. Series: Materials Science and Engineering, 451, 012211 (2018) 
7. A.V. Dmitriev, I.N. Madyshev, O.S. Dmitrieva, A.N. Nikolaev, Ecology and Industry of Russia, 21, 3, 12-15 (2017)

8. S. Altmeyer, V. Mathieu, S. Jullemier, P. Contal, N. Midoux, S. Rode, J.P. Leclers, Chem. Eng. Prog, 43, 511-522 (2004)

9. O.V. Soloveva, S.A. Solovev, R.R. Khusainov, A.S. Shubina, J. Phys.: Conf. Ser. 1158, 042023 (2019)

10. C. Wen, X.W. Cao, B. Yan, J. Zhang, Appl. Mech. Mater. 44-47, 1913-1917 (2011)

11. J. Song, X. Hu, Sep. Purif. Technol., 178, 242-252 (2017)

12. W.H. Walton, Dust Control and Air Cleaning. Cyclone dust separators (Elsevier, Amsterdam, 2014)

13. C. Cortés, A. Gil, Prog. Energy Combus.t Sci., 33, 409-452 (2007)

14. N.A. Merentsov, V.A. Balashov, S.A. Bokhan, E.E. Nefed'eva, D.A. Tezikov, V.V. Groshev, IOP Conf. Ser.: Earth Environ. Sci., 224, 012041 (2019)

15. I.N. Madyshev, O.S. Dmitrieva, A.V. Dmitriev, MATEC Web Conf., 91, 01019 (2017)

16. F. Shuanshi, X. Wang, X. Lang, Y. Wang, Nat. Gas Ind., 4(6), 470-476 (2017)

17. O.S. Makusheva, A.V. Dmitriev, N.A. Nikolaev, Chem. Pet. Eng., 46, 5-6, 330-333 (2010)

18. J.P. Tranier, Energy Procedia, 4, 966-971 (2011)

19. R. Staudt, Adsorption, 11, 3-4, 379-384 (2005)

20. A. Avci, I. Karagoz, J. Aerosol Sci., 53, 937-955 (2003) 